\title{
MIP and MEP Changed by Acupuncture Techniques in Costal
}

\section{Morais Sanchez EG, Silva DO and Sanchez HM* \\ Department of Physiotherapy, Federal University of Goias (UFG), Brasil}

*Corresponding author: Hugo Machado Sanchez, Department of Physiotherapy, Federal University of Goias (UFG), Brasil, Email: hmsfisio@yahoo.com.br

\section{Research Article \\ Volume 2 Issue 4}

Received Date: October 01, 2019

Published Date: October 23, 2019

DOI: $10.23880 /$ aphot- 16000140

\section{Abstract}

The respiratory tract is of great importance due to integrated functions of various systems, which allow gas exchange and the arrival of oxygen to the muscles. The aim of this study was to evaluate the respiratory pressure after acupuncture technique in the rib cage. Was a randomized study with 20 volunteers, age 18-25 years, divided into two groups 10 men and 10 women. The two groups were subjected to evaluation of the manometer respiratory pressures which were taken before and after treatment, physical therapy, which was used in costal acupuncture in one session of 10 minutes, during this time were made movements of rotation of the needle 5 in first minutes after application, the fifth and tenth minutes minutes after application, and then the needle was withdrawn the patient returned to the starting position, sitting where the measures were repeated maximal respiratory pressures. The values of maximal respiratory pressures (MIP and MEP) in men before and after the intervention period showed significant changes $(p<0.05)$. In women, the values maximal respiratory pressures (MIP and MEP) showed statistically significant differences after the intervention period $(\mathrm{p}<0.05)$, comparing men and women MIP before and after the intervention period, no statistically significant, since the MEP before and there was no statistical significance after the intervention period was statistically significant in PE between men and women. Whereas $\mathrm{p}<0.05$. The results of this study show statistically significant differences, since these were obtained due to the low number of patients this study can serve as props for other jobs.

Keywords: Respiratory Pressures; Acupuncture; Manometer; Diaphragm; Pressure Changes

Abbreviations: MIP: Maximal Inspiratory Pressure; MEP: Maximum Expiratory Pressure; TCM: Traditional Chinese Medicine; PNF: Proprioceptive Neuromuscular Facilitation; COPD: Chronic Obstructive Pulmonary Disease.

\section{Introduction}

The respiratory tract is of great importance due to the integrated functions of various systems, which are the possible gas exchange and the arrival of oxygen to the muscles. A muscle of great importance for breathing is the diaphragm [1].

The diaphragm may be shortened due to various incorrect postural positions adopted by the individual, which may have impairment of its function in relation to the forces exerted by it. Thus, to evaluate the respiratory muscle strength there are several ways among them the present study used the manovacuometry [2]. 


\section{Annals of Physiotherapy \& Occupational Therapy}

The manovacuometer can determine the changes in respiratory muscles with great precision, as it allows the measurement of inspiratory muscle strength and expiratory muscle strength, determined by negative pressure and positive pressure [3].

Maximum pressure assessments consist of measuring the strength of inspiratory muscles designated as maximal inspiratory pressure (MIP) and expiratory muscles termed as maximum expiratory pressure (MEP) [4].

The evaluation of maximal inspiratory and expiratory pressure values has the function in the diagnosis and prognosis of neuromuscular and pulmonary disorders, and it is important to evaluate respiratory muscle strength to quantify the progression of fatigue, muscle weakness and also allow the diagnosis of respiratory failure [2]. Caused by muscle failure, helping to assess respiratory mechanics and to indicate intubation, respirator weaning and extubation of patients [5].

Acupuncture is one of the traditional Chinese medicine (TCM) techniques used to treat energy, functional and organic imbalances. An ancient therapeutic method aimed at therapy and cure of diseases by applying stimuli through the skin, with the insertion of needles at specific points [6-8].

The skin has a high concentration of sensory nerve endings, its stimulation allows direct access to the CNS [8]. It is also a reflex therapy, where the stimulation of one area acts on another (s). For this purpose, it mainly uses nociceptive stimulation [9]. The most likely stimulus receptor sites, according to morphofunctional studies, are nerve plexuses, vascular elements, and muscle bundles [10].

Nociceptive receptor stimuli, triggered by needle insertion, generate an electrical action potential and minimal local inflammatory process. With the release of several neurotransmitter substances, encephalins and serotonins, responsible for the promotion of analgesia [11]. Acupuncture acts on the analgesic response by stimulating nerves that carry stimuli to the spinal cord, and activate the brainstem and hypothalamus neurons, thus releasing endogenous opioids, which explains the mechanisms of control of acute and chronic pain [12].

Thus, this study aims to evaluate the differences in Maximum Inspiratory Pressure (MIP) and Maximum Expiratory Pressure (MEP) in healthy young individuals undergoing costal margin acupuncture.

\section{Methods}

This study was carried out at the school clinic, in which 20 undergraduates participated in the study, 10 women and 10 men, aged between 18 and 25 years, having as inclusion criteria to be in the comprised age group; non-practitioners of sports activities; no history of previous lung disease; male and female gender; nonsmokers. As an exclusion criterion, volunteers with a history of lung diseases did not participate in the study; do not show the required age for the survey; volunteers with altered ribcage; volunteers who experience any pain during the evaluation; volunteer smokers. All signed an informed consent and the study was approved by the Research Ethics Committee.

Respiratory pressures in $\mathrm{cmH} 2 \mathrm{O}$ generated at mouth level were measured with a manovacuometer (MTR - ind. Bras.), With an operating range of 0 to $-300 \mathrm{cmH} 2 \mathrm{O}$ and 0 to $+300 \mathrm{cmH} 20$ adapted for maximal inspiratory and expiratory pressures. A plastic tubing was connected to the manovacuometer and at the distal end of the tube was fitted a cylindrical rubber mouthpiece with an internal diameter of $2.4 \mathrm{~cm}$; anterior to the mouthpiece, a $16 \mathrm{~cm}$ long plastic device was placed to provide small air leakage to prevent the elevation of the oral cavity pressure generated exclusively by contraction of the facial muscles with glottis closure [13].

All measurements of maximal respiratory pressures were collected by the same researcher performed under homogeneous verbal command. Measurements were made with the volunteers seated and their nostrils occluded by nasal tweezers to prevent air leakage. MIP was measured during exertion initiated from the residual volume -VR, while MEP was measured from total lung capacity - TLC [14]. Each volunteer performed three technically satisfactory maximum inspiratory and expiratory efforts, ie, without perioral air leakage and with values close to each other, being considered for the study the highest value measure [15].

After collecting the pressures, acupuncture was performed on the costal margin, the researcher wore disposable gloves and in each volunteer was made aseptic with cotton and alcohol in place before application. After this procedure, the volunteers were instructed to lie supine on a platform, 1.4 meters wide and 2.0 meters long, and in this position 7 needles were placed on the costal margin from the xiphoid region. to the anterior end of the last rib on either side of the abdominal region. Sterile disposable capillary acupuncture needles of 0.10 to $0.50 \mathrm{~mm}$ in diameter and 1.5 to $10 \mathrm{~cm}$ in length were 
used. The insertion of the acupuncture needle was made by holding with the left hand the mandrel which is rigid tubular device in which the needle will be placed to guide the depth of penetration of $3 \mathrm{~mm}$ of the needle into the skin, with the right hand the needle was introduced with the rapid bending movement of the indicator over the needle.

To avoid risk of perforation of structures under the skin, the needle inclination was 45 degrees, ie, an oblique puncture [16]. After needle insertion, a piston movement was made in the needle so that more skin receptors could be stimulated. The needle was 10 minutes in place and during this time 5-minute movements of the needle were made in the first minute after application, in the fifth and tenth minutes after application, and then the needle was removed and again cleaned with cotton and alcohol.

After the removal of the needles, the patient returned to the initial sitting position, where the maximum respiratory pressures were measured again.

\section{Results}

The MIP and MEP values obtained before acupuncture and after acupuncture showed significant differences. For statistics we used the Wilcoxon test. Tables 1, 2 and Figures $1 \& 2$ describe the absolute and relative values in relation to the predicted MIP and MEP values obtained before and after the intervention period, where they showed significant differences.

\begin{tabular}{|c|c|c|c|c|c|}
\hline \multirow{2}{*}{$\begin{array}{c}\text { Maximal respiratory } \\
\text { pressures }\end{array}$} & Found values & Found values & Predicted Values & Predicted Values & \multirow{2}{*}{ P value } \\
\cline { 2 - 5 } & Before & After & Before & After & \\
\hline \multicolumn{7}{|c|}{ MIP (cm $\left.\mathbf{H}_{\mathbf{2}} \mathbf{0}\right)$} \\
\hline Woman & 80,50 & 97,67 & 11,19 & 5,28 & 0,010 \\
\hline Mens & 71,67 & 91,83 & 2,04 & 7,03 & 0,030 \\
\hline Woman & 63,17 & 75,00 & 4,32 & 5,67 & 0,000 \\
\hline Mens & 71,83 & 90,67 & 3,79 & 5,37 & 0,000 \\
\hline
\end{tabular}

Table 1: Maximal inspiratory pressure (MIP) and maximum Expiratoria (MEP) values. Data are expressed as Media \pm standard deviation, * statistical significance $(\mathrm{P}<0.05)$, using Wilcoxon's statistical tests.

\begin{tabular}{|c|c|c|}
\hline Analyzed Variables & Comparision & Pressure Values \\
\hline MIP before & Men X Woman & 0,721 \\
\hline MIP after & Men X Woman & 0,914 \\
\hline MEP before & Men X Woman & 0,112 \\
\hline MEP after & Men X Woman & 0,013 \\
\hline
\end{tabular}

Table 2: Analysis of MIP and MEP before and after the costal margin acupuncture technique in men and women.



Sanchez HM, et al. MIP and MEP Changed by Acupuncture Techniques in Costal. Ann Physiother Occup Ther 2019, 2(4): 000140. 


\section{Annals of Physiotherapy \& Occupational Therapy}

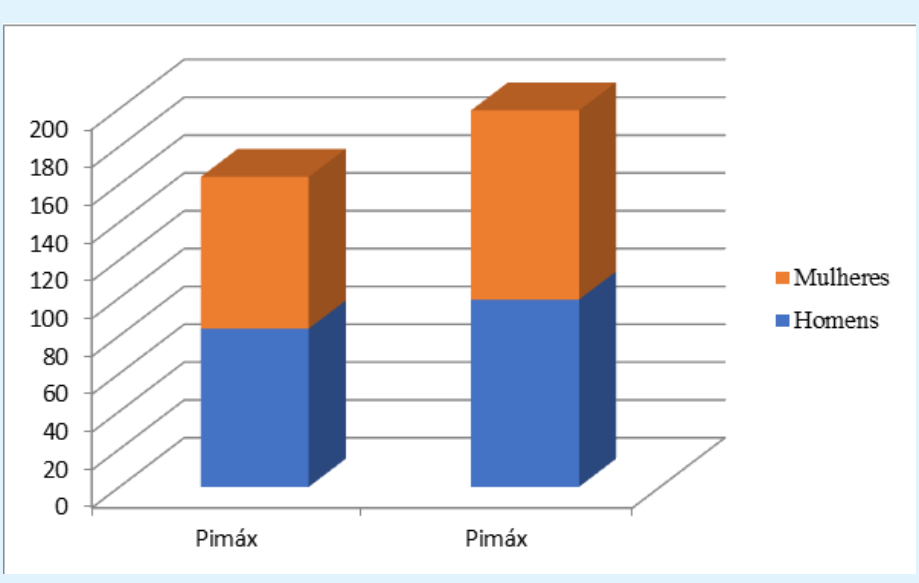

Figure 2: MEP before and after acupuncture technique in men and women.

\section{Discussion}

The inspiratory muscles are shortened due to the displacement of the equilibrium position of the respiratory system, there is a decrease in expired air and changes in the ability to generate volume changes, thus finding less effective air volume for gas exchange. . A muscle of great importance for breathing is the diaphragm [15].

The diaphragm may be shortened due to various incorrect postural positions adopted by the individual, in which its function may be compromised in relation to the forces exerted by it. Thus, to assess respiratory muscle strength, there are several ways, including manovacuometry [2].

Through the manovacuometer it is possible to determine with great precision the alterations in the respiratory musculature, since it allows the measurement of the inspiratory muscles strength and the expiratory muscles strength, determined by negative pressure and positive pressure [3].

The measurement of the maximum respiratory pressures generated during respiratory efforts represents a very useful procedure for the functional evaluation of the respiratory muscles involved in ventilation [15]. It is important to assess respiratory muscle strength to quantify the progression of fatigue, muscle weakness and to identify neuromuscular diseases [1].

Acupuncture is used to treat energy, functional and organic imbalances [6-8]. For this purpose, it mainly uses nociceptive stimulation [9]. Nociceptive receptor stimuli, triggered by needle insertion, generate an electrical action potential and a minimal local inflammatory process [10].

The present study demonstrated that the physical therapy intervention by the acupuncture technique applied during 1 10-minute session on young, healthy and sedentary volunteers was able to significantly increase the maximal respiratory pressure values.

Other studies have shown concern with respiratory function. Like the study by Moreno 2000 that observed increased inspiratory and expiratory muscle strength and thoracic mobility in young women, sedentary age $22.65 \pm$ 2.5 years after 4 weeks of training using proprioceptive neuromuscular facilitation (PNF) techniques. ), which collaborates with the present study, which investigated pressure changes in young people using the costal margin acupuncture technique where the results were satisfactory in relation to the increase in inspiratory and expiratory pressures at such times before and after [17].

Piaia and Moreno [13] report that the values of respiratory pressures are influenced by body position. These authors verified higher values in the pressures in the sitting position in relation to the supine position and report that it is due to the favoring of diaphragmatic mechanics. Collaborating with the present study in which measurements were taken in the sitting position, which were similarly increased [13].

Teodori and Moreno [18] conducted a study evaluating the pressures after a single RPG intervention session in 20 healthy young women. The MIP showed a statistically significant increase that was attributed to increased respiratory muscle strength and mobility. Chest 


\section{Annals of Physiotherapy \& Occupational Therapy}

as an effect of stretching, which involved the respiratory muscle chain in global posture [18]. The present study did not use postural treatment, but the direct intervention in the diaphragmatic region with the use of acupuncture and increased pressure in the same way, being the technique of great effectiveness to improve respiratory function and may contribute to more efficient treatments.

This research aimed to study the changes in respiratory pressures by the acupuncture technique on the diaphragmatic region, to support future studies involving individuals with respiratory dysfunction.

Godoy and Bringhenti [19] observed the influence of generalized muscle weakness and the mechanical disadvantage of inspiratory muscles on MIP attainment in dpoc patients and found an increase in MIP after stretching, which is also in agreement with the present study, which suggests improvement. on the mechanical advantage of the diaphragm [19].

Paulin and Brunetto [20] the increase in lung volume is reflected in a significant shortening of inspiratory muscles. This increase is mainly present in obstructive respiratory diseases, such as chronic obstructive pulmonary disease (COPD). Thus, the present study may support pathological treatments for patients.

\section{Conclusion}

Although the study presented data related to healthy people, the results achieved indicate a probable improvement in diaphragmatic function, contributing to the increase in respiratory pressures evidenced in the present research. Acupuncture in the costal margin may be an important therapeutic resource in the treatment of respiratory muscle dysfunction.

Therefore, further studies are needed to evaluate the effect of acupuncture on increasing respiratory pressures in a larger number of healthy volunteers.

\section{References}

1. Machado, Rodrigues GMD (2008) Bases of Respiratory Physiotherapy-Intensive Care and Rehabilitation. Rio de Janeiro: Guanabara, Koogan.

2. Ires M (1999) Fisiologia. Rio de Janeiro: Guanabara, Koogan.
3. Guyton AC, Hall JE (2006) Treatise on Medical Physiology, $11^{\text {th }}$ (Ed.), Rio de Janeiro, Elsevier Editora, LTDA.

4. Silverthorn DU, William C Ober (2003) Fisiologia Humana: Uma abordagem integrada. Sao Paulo, Manole.

5. Cingalani HE, Houssay AB (2004) Fisiologia Humana. Porto Alegre, Artmed.

6. wen TS (1989) Acupuntura classica chinesa. $2^{\text {nd }}($ Ed.), Sao Paulo, pp: 225.

7. Jaggar D (1992) History and basic introduction to veterinary acupuncture. Problems in Veterinary Medicine 4(1): 1-11.

8. Schoen AM (1993) Introduction to veterinary acupuncture: scientific basis and clinical aplications. In: Annualconvention of The American Association ofequine Practioners California. California, pp: 39.

9. Farber PL, Timo-Iaria C (1994) Acupuntura e sistema nervoso. Jornal Brasileiro de Medicina 67(5-6): 125131.

10. Lundeberg $\mathrm{T}$ (1993) Peripheral effects of sensory nerve stimulation (acupuncture) in inflammation and ischemia. Scand J Rehabil Med 29: 61-86.

11. Wink S, Cartana, Maria do HF (2007) Promoting selfcare for headache patients through the oriental health perspective. Rev Bras Nursi 60(2): 225-228,

12. Hopwood Val, Lovesey M, Mokone S (2001) Acupuncture and Physiotherapy Related Techniques, pp: 245.

13. Piaia IM, Moreno MA (2002) A influência da postura na força muscular respiratória. Rev Bras Fisiot, pp: 45.

14. Moreno G, Marlene A (2000) Proprioceptive neuromuscular facilitation Patterns and its effectual respiratory capacity. Postgraduate Program in Oral and Dental Biology and Pathology, pp: 237.

15. Camelo JSJ, Terra JF, Manco JC (1985) Maximal respiratory pressures in normal adults. J Pulmonology 11(4): 181-184.

16. Yamamura Y (2001) Traditional Acupuncture: The Art of Inserting, pp: 919. 
17. Moreno G, Marlene A (2000) Proprioceptive neuromuscular facilitation patterns and their effect on respiratory capacity. Dissertation (masters) - State University of Campinas, pp: 237.

18. Teodori RM, Moreno MA, Fiori Junior JF, Oliveira ACS (2003) Inspiratory Muscle Stretching Through Global Postural Reeduction. Rev Bras Fisioter 7(1): 25-30.
19. Godoy DV, Bringhenti RL, Severa A, Gasperi R, Poli LV, et al. (2006) Yoga versus aerobic activity: effects on spirometric tests and maximal inspiratory pressure. J Bras Pneumol 32(2): 130-135.

20. Paulin E, Brunetto AF, Carvalho CRF (2003) Effects of a physical exercise program aimed at increasing thoracic mobility in patients with chronic obstructive pulmonary dis ease. J Pneumol 29(5): 287-294. 